\title{
Analysis of diabetes quality assessment findings and future directions for the appropriate manage- ment of diabetes in Korea
}

Yu Jin $\mathrm{Kim}^{1}$, Suk Chon ${ }^{2}$, Seungjoon $\mathrm{Oh}^{2}$, Jeong-taek Woo ${ }^{2}$, Sung Woon $\mathrm{Kim}^{2}$, and Sang Youl Rhee ${ }^{2}$

\begin{abstract}
${ }^{1}$ Department of Medicine, Graduate School, Kyung Hee University, Seoul; ${ }^{2}$ Department of Endocrinology and Metabolism, Kyung Hee University School of Medicine, Seoul, Korea
\end{abstract}

Received: April 25, 2016 Revised : August 23, 2016 Accepted: October 27, 2016

\author{
Correspondence to \\ Sang Youl Rhee, M.D. \\ Department of Endocrinology \\ and Metabolism, Kyung Hee \\ University School of Medicine, \\ 23 Kyungheedae-ro, Dongdae- \\ mun-gu, Seoul 02447, Korea \\ Tel: +82-2-958-8200 \\ Fax: +82-2-968-1848 \\ E-mail: bard95@hanmail.net
}

Background/Aims: Due to recent increases in the disease burden of diabetes mellitus, the Health Insurance Review and Assessment Service (HIRA) of Korea implemented a quality assessment of the treatment of diabetes to improve patient care. The present study was conducted to identify any changes after the implementation of the diabetes quality assessment (DQA).

Methods: The present study evaluated eight quality assessment indicators that were proposed by the HIRA in all patients with diabetes who visited a university hospital in Korea between 2009 and 2014. The indicators were statistically compared according to the characteristics of the subjects.

Results: There were several significant differences in the indicators among the subjects according to their demographic characteristics. Female patients had a higher continuity of treatment (COT) than that of male patients, and the insulin-treated group had a higher COT than that of the non-treated group, as well as a higher rate of undergoing the diabetes complication tests (DCTs). Patients between 40 and 80 years of age had the highest COT, while patients under 40 years of age had the lowest COT but the highest rate of taking the DCTs. Patients receiving treatment from an endocrinologist exhibited higher numbers of DCTs performed but displayed lower proportions for the prescription indicators.

Conclusions: The present analysis of the DQA findings revealed that endocrinologists combine prevention and management of diabetes complications with measures for glycemic control. Thus, the effective management of diabetes likely entails systematic joint treatment regimens that involve an endocrinologist.

Keywords: Diabetes mellitus; Quality of health care; Quality improvement; Diabetes complications; Endocrinology

\section{INTRODUCTION}

In 2011, the prevalence of diabetes mellitus in Korea was $7.7 \%$, which is higher than the average prevalence of $6.9 \%$ reported by the Organization for Economic Co-operation and Development (OECD) [1]. During that same period, the hospitalization rate in Korea due to diabe- tes was 29 per 1,000 individuals, which was also higher than the average rate reported by the OECD of 23.8 per 1,000 individuals. In 2013, diabetes was ranked as the fifth leading cause of death in Korea [2], and the number of diabetic patients and cost of diabetes-related medical expenses are consistently increasing [3]. As of 2013, approximately 2.54 million patients with diabetes visited 
outpatient units for treatment, which represents a 1.98\% increase from the 2.49 million patients who visited outpatient units in 2012 [4]. Moreover, outpatient medical expenses totaled approximately 370 million KRW (Korean won) in 2013, which was an increase of $8.01 \%$ from the 340 million KRW spent on medical expenses in 2012.

A number of previous studies have suggested that appropriate care of patients with diabetes would significantly reduce complications related to this disease. For example, intensive glucose control reduces the risk of any cardiovascular disease (CVD) event by $42 \%$ and the risk of heart attack, stroke, or death due to CVD by $57 \%$ [5]. Stratton et al. [6] found that a $1 \%$ reduction in mean glycated hemoglobin (HbAic) levels results in $21 \%$ fewer deaths, $14 \%$ fewer myocardial infarctions, and a $37 \%$ decrease in microvascular complications at the population level. Similarly, a report from the US Department of Health and Human Services indicated that improved control of levels of cholesterol or blood lipids, including high density lipoprotein cholesterol, low density lipoprotein (LDL) cholesterol, and triglycerides, reduces CVD complications by $20 \%$ to $50 \%$ [7]. Furthermore, the detection and treatment of diabetic eye disease using laser therapy reduces the development of severe vision loss by an estimated 50\% to $60 \%$, while the detection and treatment of early diabetic kidney disease through the lowering of blood pressure levels attenuates the decline in kidney function by $30 \%$ to $70 \%$ [7].

As a countermeasure to the continuously increasing number of patients with diabetes and the concomitant increase in medical expenses, a model for the quality assessment of diabetes treatment in Korea was developed to reduce the risk of diabetes complications and enhance the adequacy of medical expenses through improvements in the management of diabetic patients. The diabetes quality assessment (DQA) was implemented by the Health Insurance Review and Assessment Service (HIRA) of Korea in 2011 and has been ongoing since its induction. The present study aimed to identify the management statuses of domestic diabetic patients and to predict future trends in diabetes treatment by assessing the DQA indicators of diabetic patients who received treatments at a university hospital in Korea between 2009 and 2014. The data were compared and analyzed according to the demographic characteristics of the patients.

\section{METHODS}

\section{Diabetes quality assessment}

The DQA has been implemented in all medical institutions that submit medical expense claims for diabetes as either a main or secondary disease and is administered during annual outpatient treatment visits. Medical bills claimed by healthcare providers, including diabetes as disease and the prescription of hypoglycemic agents, are used as the assessment data [4]. The DQA has implemented in Korea since 2011, and all indicators identified from health insurance claims are analyzed; the assessment and monitoring indicators currently in use are described in Table 1.

The assessment indicators analyzed in the present study included the following: the proportion of patients who visited a center more than once per quarter and the proportion of prescription days corresponding to continuity of treatment (COT), the proportions of duplicated prescriptions involving the same ingredient groups and prescriptions with over four ingredient groups corresponding to prescription indicators, and the proportion of patients who underwent $\mathrm{HbAcc}$, lipid, and fundus tests corresponding to test indicators. High scores for the COT and test indicators suggest good diabetes management, while low scores for the prescription indicators were considered optimal [4]. The monitoring indicators analyzed in the present study are as follows: the proportion of patients who underwent microalbuminuria tests corresponds to a test indicator, and the medication cost per day for prescribed hypoglycemic agents and the proportion of combined prescriptions that do not adhere to the criteria correspond to prescription indicators. All of the abovementioned variables are considered process indicators, while outcome indicators, such as the management of lifestyle and the HbAic control [8], will be phased in the near future by HIRA [9].

\section{Subjects and materials}

The Kyung Hee University Hospital implemented its own DQA indicator management system, and the assessment and monitoring indicators used in the present study were based the diabetic patients who have been treated in this hospital since 2009. The structure of the DQA indicator management system used at Kyung Hee University Hospital is presented in Supplementary 


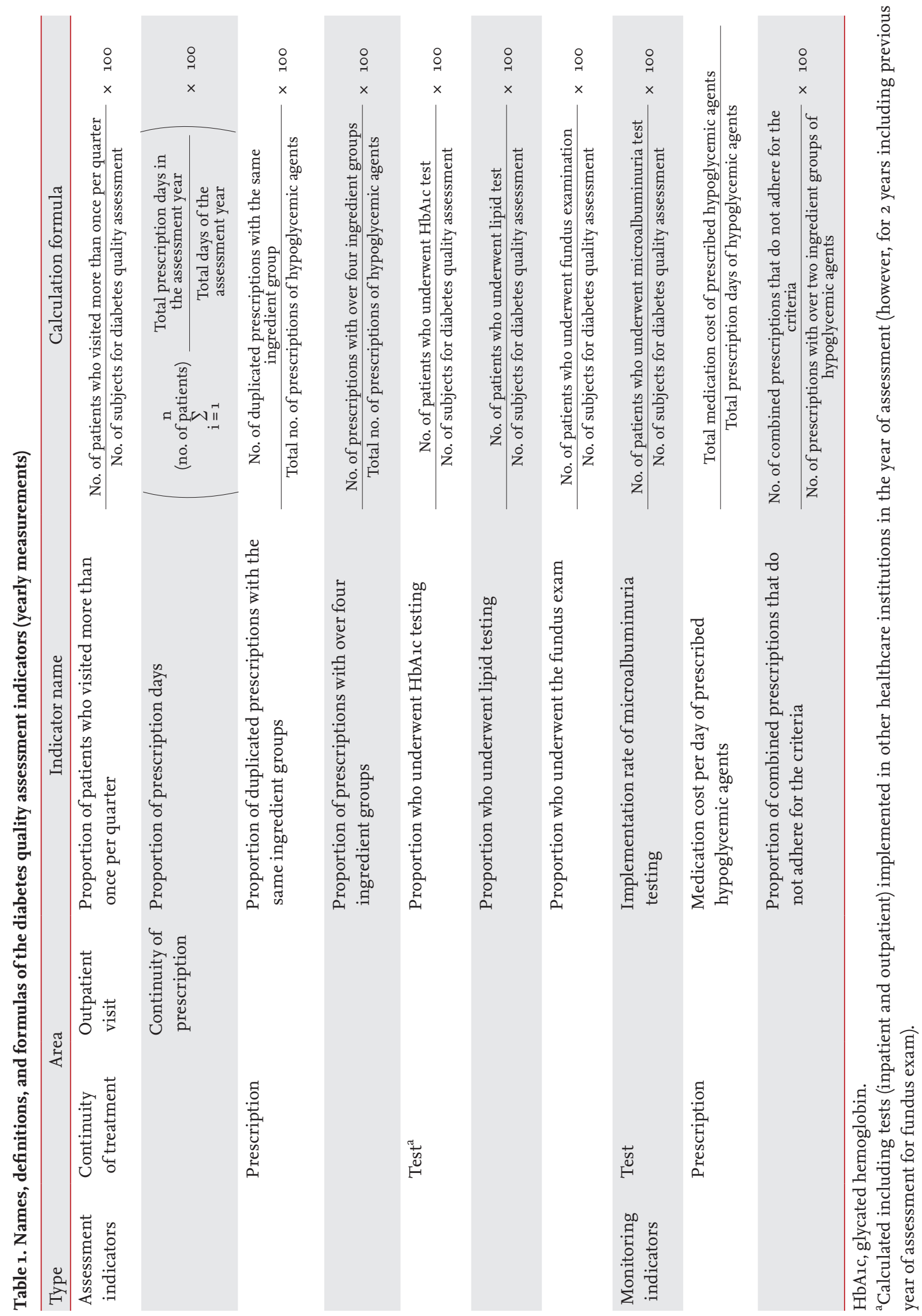


Fig. 1. The indicators were classified into three primary categories: continuity, test, and prescription indicators. The proportion of patients who visited the center more than once per quarter and the proportion of prescription days are included in the continuity category; the HbAıc, lipid, fundus, and microalbuminuria tests are included in the test category; and prescriptions with more than four ingredient groups, duplicated prescriptions with the same ingredient groups, and the cost per day for prescribed hypoglycemic agents are included in the prescription category. Each indicator was classified according to patient variables such as the department of treatment, sex, age, use of insulin, and duration of use of oral hypoglycemic agents and insulin and have been categorized by year. Additionally, the electronic prescription program used to manage the outpatient care of the diabetic patients includes a notification window function that alerts physicians regarding test items for which 1 year has passed since the previous diabetes complication tests (DCTs). Using this function, the physician in charge is alerted of the tests that need to be performed for each patient (Supplementary Fig. 2).

The present study analyzed data from the DQA indicator management system of Kyung Hee University Hospital to determine differences and trends in the indicators that are likely dependent on the sex and/or age of diabetic patients, their use of insulin, and the treatment department. The cost per day of prescribed hypoglycemic agents and the proportion of combined prescriptions not adhering for the criteria, which were monitoring indicators, were excluded from this analysis.

\section{Ethics statement}

Because the present study analyzed existing data from a DQA indicator management system without registering patients or contacting new patients, investigators only viewed data that did not include any personal information identifying the patients. Thus, this study was exempted from the requirement for patient consent by the Institutional Review Board prior to commencement of the study (KMC-IRB 1605-04).

\section{Statistical analysis}

All statistical analyses of the DQA indicator data were performed using SPSS version 18.0 (SPSS Inc., Chicago, IL, USA). Differences in the indicators according to the sex and age of the diabetic patients, their use of insulin, and the treatment department were analyzed using chisquare analyses. A $p<0.05$ was considered to indicate statistical significance, and a Bonferroni correction was performed for the post hoc analyses of the cross analysis.

\section{RESULTS}

From 2009 to 2014, the DQA indicators of an annual average of 8,327 patients were assessed in the present study. There were $1.31 \%$ more male than female patients, $22.76 \%$ of the total patients used insulin, and most patients were between 60 and 70 years of age. Patients who were treated by an endocrinology specialist through the Department of Endocrinology (endo group) only or in conjunction with other departments (endo \& others group) accounted for $60 \%$ of the total population, while $40 \%$ of patients were treated in departments other than endocrinology (others group). Patients who had been medicated for over 6 years accounted for $40 \%$ to $50 \%$ of the population (Supplementary Table 1).

\section{DQA indicators according to sex}

Tables 2-4 display the assessment indicators according to sex and insulin use. In terms of COT, the proportion of patients who visited the center more than once per quarter was higher among female patients in all years, which indicates that female patients visited the outpatient unit more consistently than did male patients. The proportion of prescription days was also higher in female patients, which suggests that female patients were taking their prescribed medications more consistently than were male patients. In the prescription category, there were no sex-dependent differences.

\section{DQA indicators according to insulin use}

A significantly higher proportion of patients visited the center more than once per quarter in the insulin-tre ated group (yes) than non-insulin-treated group (no) in all years. The proportion of prescription days was calculated as the number of days in the year that oral hypoglycemic agents were prescribed and was lower in the insulin-treated than non-insulin-treated group. The proportion of prescriptions with over four ingredient groups was higher in the insulin-treated group, 
Table 2. Diabetes quality assessment indicators according to sex and use of insulin (continuity of treatment)

\begin{tabular}{|c|c|c|c|c|c|c|c|c|c|c|c|c|}
\hline \multirow{3}{*}{ Year } & \multicolumn{6}{|c|}{ Quarterly visit } & \multicolumn{6}{|c|}{ Proportion of prescriptions per day } \\
\hline & \multicolumn{3}{|c|}{ Sex } & \multicolumn{3}{|c|}{ Insulin } & \multicolumn{3}{|c|}{ Sex } & \multicolumn{3}{|c|}{ Insulin } \\
\hline & Male, \% & Female, \% & $p$ value & Yes, \% & No, \% & $p$ value & Male, \% & Female, \% & $p$ value & Yes, \% & No, \% & $p$ value \\
\hline 2009 & 78.7 & 82.4 & $<0.001$ & 85.4 & 79.0 & $<0.001$ & 84.6 & 85.4 & $<0.001$ & 78.7 & 86.5 & $<0.001$ \\
\hline 2010 & 80.0 & 82.2 & 0.012 & 84.6 & 80.0 & $<0.001$ & 85.9 & 85.2 & $<0.001$ & 79.6 & 87.0 & $<0.001$ \\
\hline 2011 & $74 \cdot 3$ & 78.7 & $<0.001$ & 85.0 & 73.9 & $<0.001$ & 87.5 & 88.2 & $<0.001$ & 83.1 & 88.9 & $<0.001$ \\
\hline 2012 & 74.7 & 77.8 & $<0.001$ & 82.6 & $74 \cdot 3$ & $<0.001$ & 86.9 & 86.9 & 0.909 & $79 \cdot 7$ & 88.6 & $<0.001$ \\
\hline 2013 & $75 \cdot 5$ & 78.9 & $<0.001$ & 84.5 & 75.1 & $<0.001$ & 88.3 & 87.8 & $<0.001$ & $83 \cdot 4$ & 89.1 & $<0.001$ \\
\hline 2014 & $74 \cdot 7$ & 78.5 & $<0.001$ & 82.5 & 75.0 & $<0.001$ & 86.1 & 87.1 & $<0.001$ & 82.6 & 87.4 & $<0.001$ \\
\hline
\end{tabular}

Statistics were analyzed using a chi-square test.

Table 3. Diabetes quality assessment indicators according to sex and use of insulin (prescription)

\begin{tabular}{|c|c|c|c|c|c|c|c|c|c|c|c|c|}
\hline \multirow{3}{*}{ Year } & \multicolumn{6}{|c|}{ Over four ingredient groups } & \multicolumn{6}{|c|}{ Same ingredient groups } \\
\hline & \multicolumn{3}{|c|}{ Sex } & \multicolumn{3}{|c|}{ Insulin } & \multicolumn{3}{|c|}{ Sex } & \multicolumn{3}{|c|}{ Insulin } \\
\hline & Male, \% & Female, \% & $p$ value & Yes, \% & No, \% & $p$ value & Male, \% & Female, $\%$ & $p$ value & Yes, \% & No, $\%$ & $p$ value \\
\hline 2009 & 1.17 & 1.08 & 0.371 & 2.51 & 0.58 & $<0.001$ & 0.20 & 0.04 & $<0.001$ & 0.02 & 0.16 & $<0.001$ \\
\hline 2010 & 1.21 & 1.01 & 0.032 & 1.96 & 0.79 & $<0.001$ & 0.15 & 0.06 & 0.004 & 0.02 & 0.14 & 0.001 \\
\hline 2011 & 0.45 & 0.42 & 0.560 & 0.73 & 0.33 & $<0.001$ & 0.10 & 0.06 & 0.102 & 0.03 & 0.10 & 0.021 \\
\hline 2012 & 0.52 & 0.35 & 0.006 & 0.89 & 0.26 & $<0.001$ & 0.14 & 0.07 & 0.019 & 0.02 & 0.13 & 0.002 \\
\hline 2013 & 0.79 & 0.68 & 0.189 & 1.88 & 0.36 & $<0.001$ & 0.11 & 0.10 & 0.664 & 0.00 & 0.14 & $<0.001$ \\
\hline 2014 & 1.18 & 0.74 & $<0.001$ & 2.14 & 0.59 & $<0.001$ & 0.15 & 0.11 & 0.292 & 0.03 & 0.17 & 0.001 \\
\hline
\end{tabular}

Statistics were analyzed using a chi-square test.

Table 4. Diabetes quality assessment indicators according to use of insulin (test)

\begin{tabular}{|c|c|c|c|c|c|c|c|c|c|c|c|c|}
\hline \multirow{2}{*}{ Year } & \multicolumn{3}{|c|}{ HbAic } & \multicolumn{3}{|c|}{ Lipid } & \multicolumn{3}{|c|}{ Fundoscopy } & \multicolumn{3}{|c|}{ Microalbumin } \\
\hline & Yes, \% & No, $\%$ & $p$ value & Yes, \% & No, $\%$ & $p$ value & Yes, \% & No, $\%$ & $p$ value & Yes, \% & No, $\%$ & $p$ value \\
\hline 2009 & 97.2 & 91.9 & $<0.001$ & 84.7 & 80.2 & $<0.001$ & 49.0 & 27.0 & $<0.001$ & 43.0 & 28.6 & $<0.001$ \\
\hline 2010 & 97.4 & $93 \cdot 5$ & $<0.001$ & $84 \cdot 3$ & 81.8 & 0.012 & $47 \cdot 3$ & 27.9 & $<0.001$ & 41.6 & 27.5 & $<0.001$ \\
\hline 2011 & 97.6 & $94 \cdot 5$ & $<0.001$ & 88.7 & 85.4 & $<0.001$ & 64.5 & 40.5 & $<0.001$ & 72.1 & 48.2 & $<0.001$ \\
\hline 2012 & 98.0 & $94 \cdot 3$ & $<0.001$ & 89.7 & 85.9 & $<0.001$ & 52.7 & 31.2 & $<0.001$ & 78.6 & $53 \cdot 5$ & $<0.001$ \\
\hline 2013 & 98.1 & 94.6 & $<0.001$ & 87.0 & 84.0 & 0.001 & $53 \cdot 9$ & 32.9 & $<0.001$ & 71.4 & $47 \cdot 7$ & $<0.001$ \\
\hline 2014 & 98.4 & 95.2 & $<0.001$ & 83.2 & 82.9 & 0.732 & 58.4 & 36.1 & $<0.001$ & 70.8 & 47.1 & $<0.001$ \\
\hline
\end{tabular}

Statistics were analyzed using a chi-square test.

HbAic, glycated hemoglobin.

which likely was due to the fact that insulin is normally prescribed to patients who do not have sufficient glycemic control despite combined therapy using oral hypoglycemic agents with over three ingredient groups. On the other hand, the proportion of duplicated prescrip- tions with the same ingredient group was higher in the non-insulin-treated group. In the test category, the proportions of patients who underwent HbAlc, lipid, fundoscopy, and microalbuminuria tests were higher in the insulin-treated group. 


\section{DQA indicators according to age}

The proportion of patients who visited the center more than once per quarter was higher among patients 40 to 80 years of age (Fig. 1A), and accordingly, the proportion of prescription days was also higher in this age group (Fig. 1B). An analysis of the Korean patients included in the 2011 DQA revealed that, although the average COT (proportion of patients visiting more than once per quarter and proportion of prescription days) for all diabetic patients was over $85 \%$, the group of patients under 40 years of age had a COT of $60 \%$ to $70 \%$ [10]. This finding is consistent with the assessment results from this university that patients under 40 years of age displayed the lowest proportions of patients visiting more than once per quarter and prescription days among the three age groups.

For the test items (Fig. $1 \mathrm{C}-1 \mathrm{~F}$ ), the under 40 years age group had the lowest COT but the highest proportion of patients who underwent $\mathrm{HbArc}$, lipid, fundoscopy, and microalbuminuria tests among the three age groups. There were no statistical differences between the under 40 and 40 to 80 years age groups in terms of the proportions of HbAlc, lipid, and fundoscopy tests, but there was a difference in the proportion of microalbuminuria examinations performed. The age group over 80 years had difficulty visiting the hospital regularly and, thus, had a lower proportion of patients took the DCT. The proportion of patients who took the test significantly differed from those in the under 40 and 40 to 80 years age groups. No significant differences were observed among the age groups in the prescription category (Fig. ${ }_{1} \mathrm{G}$ and $\left.{ }_{1} \mathrm{H}\right)$.

\section{DQA indicators according to treatment department}

The proportion of patients who visited the center more than once per quarter (Fig. 2A) was highest in the endo \& others group but was not significantly differ from that in the endo group. The proportion of prescription days (Fig. 2B) was higher in the endo than endo \& others group, which also included a higher proportion of patients visiting more than once per quarter. In the test category (Fig. $2 \mathrm{C}$ and $2 \mathrm{D}$ ), there were no differences between the endo and endo \& others groups in terms of the proportion of patient who underwent the $\mathrm{HbAlc}$ and lipid tests, but the proportions in each of these groups significantly differed from that of the others group. Ad- ditionally, a trend analysis revealed that the changes in the proportion of tests performed after implementation of the DQA were small in those treated in the Endocrinology Department (endo and endo \& others groups), where the HbAıc and lipid tests had already been conducted, while the proportions of these tests performed were increased gradually among those treated in other departments (other); from 2009 to 2014 , the proportions of patients who underwent the HbAic test were 84.6\%, 87.4\%, 88.9\%, 89.1\%, 90.05\%, and 91.1\%; $p$ for trend < 0.001 , respectively, and who underwent the lipid test were $68.7 \%$, 70.1\%, 74.2\%, 74.5\%, 74.5\%, 75.0\%, and 73.0\%; $p$ for trend $<0.001$, respectively.

The proportion who underwent fundoscopy testing was highest in the endo \& others group (Fig. 2E), while the proportion of microalbuminuria examinations performed was highest in the endo group (Fig. 2F). In the others group, the proportions of $\mathrm{HbAic}$, lipid profile, fundoscopy, and microalbuminuria tests performed were lower than the national average in tertiary hospitals (indicated by solid and dotted lines) as well those in the endo and endo \& others groups; these differences were statistically significant.

The proportion of prescriptions with over four ingredient groups and the proportion of duplicated prescriptions with the same ingredient groups both corresponded to the prescription category, in which lower scores indicated better management of diabetic patients (Fig. $2 \mathrm{G}$ and $2 \mathrm{H}$ ). These proportions were low in the endo group but high in the others group. In other words, patients treated by an endocrinology specialist with endocrinology-only treatment or endocrinology treatment in conjunction with other departments showed higher proportions of prescription days and of DCTs performed but lower proportions of prescriptions with the same ingredient group and prescriptions with over four ingredient groups.

\section{DISCUSSION}

It has been estimated that the prevalence of diabetes among all age groups worldwide will increase from $2.8 \%$ in 2000 to $4.4 \%$ in 2030 , because the total number of people with diabetes is projected to rise from 171 million to 366 million during the same period [11]. This 

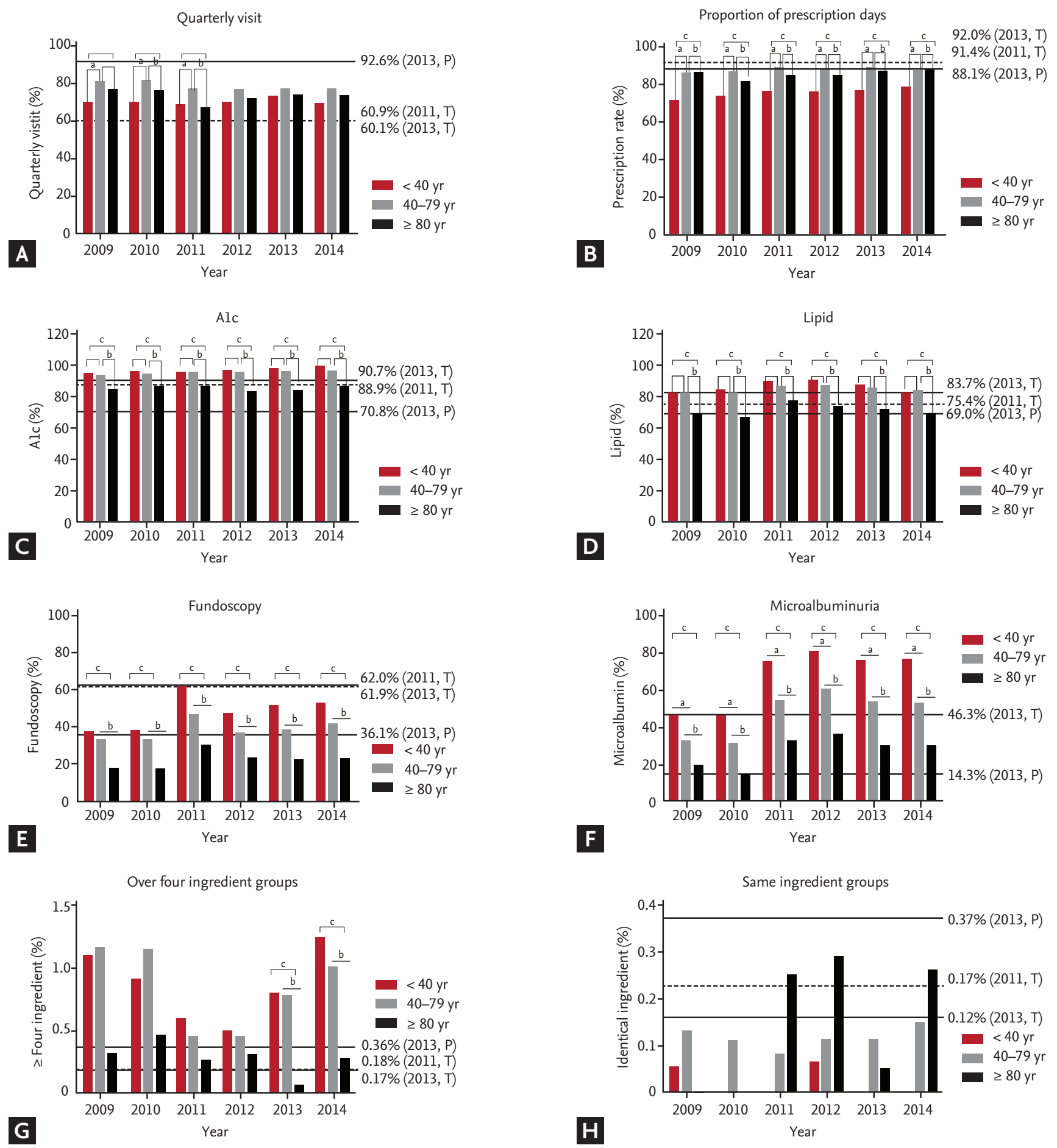

Figure 1. Differences in the annual diabetes quality assessment indicators according to age group. (A) Proportion of patients visiting more than once per quarter and (B) proportion of prescription days. Proportions of patients who underwent the (C) glycated hemoglobin (HbAic), (D) lipid, (E) fundoscopy, and (F) microalbuminuria tests. (G) Proportion of prescriptions with over four ingredient groups and $(\mathrm{H})$ the proportion of duplicated prescriptions with the same ingredient group (Solid and dotted lines indicate the mean values of the diabetes indicators at tertiary hospitals and clinics in 2013 and tertiary hospitals in 2011, respectively. Because the microalbuminuria test was introduced in 2013, there are no 2011 data). All data were analyzed using the chi-square test, and post hoc analyses were performed using Bonferroni's correction. T, tertiary hospitals; P, primary

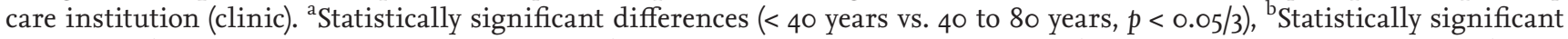
differences $\left(40\right.$ to 80 years vs. $>80$ years, $p<0.05 / 3$ ), ${ }^{c}$ Statistically significant differences $(<40$ years vs. $>80$ years, $p<0.05 / 3$ ). 

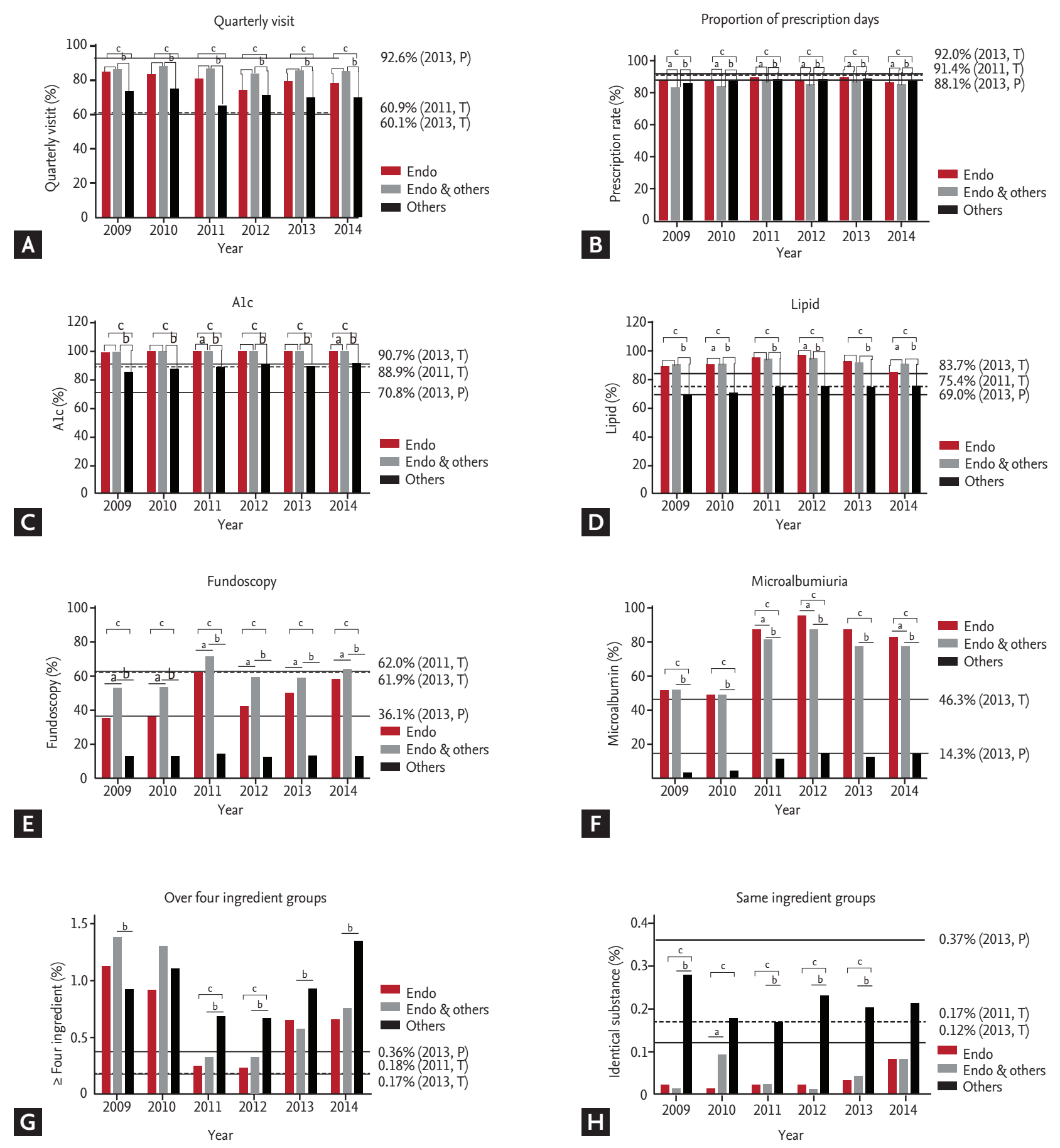

Figure 2. Differences in the annual diabetes quality assessment indicators according to the treatment department. (A) Proportion of patients visiting more than once per quarter. This indicator was the highest (B) Proportion of prescription days. Proportions of patients who underwent the (C) glycated hemoglobin (HbArc), (D) lipid, (E) fundoscopy and (F) microalbuminuria tests. (G) Proportion of prescriptions with over four ingredient groups and $(\mathrm{H})$ the proportion of prescriptions with the same ingredient group (Solid and dotted lines indicate the mean values of the diabetes indicators at tertiary hospitals and clinics in 2013 and tertiary hospitals in 2011, respectively. Because the microalbuminuria test was introduced in 2013, there are no 2011 data). All data were analyzed using the chi-square test, and post hoc analyses were performed using Bonferroni's correction. T, tertiary hospitals; P, primary care institution (clinic). ${ }^{a}$ Statistically significant differences (endo vs. endo \& others, $p<0.05 / 3$ ), ${ }^{\mathrm{b}}$ Statistically significant differences (endo \& others vs. others, $p<0.05 / 3$ ), ${ }^{\mathrm{C}}$ Statistically significant differences (endo vs. others, $p$ $<0.05 / 3)$. 
increase has clinical relevance in adults because diabetes is a primary cause of blindness [12], non-traumatic lower-limb amputation [13], and kidney failure that requires transplantation and dialysis [14]. Therefore, prevention, early detection, and treatment of the complications of diabetes as well as glycemic control need to be considered for the management of patients with this disease.

Because the prevalence of diabetes and the resulting disease burden are rising, the interest in more effective techniques for the management of diabetes has increased. Additionally, attempts to improve the quality of diabetes care have led to high quality and guideline-driven care for every diabetic patient. Thus, an accurate assessment of the level of diabetes care necessary for each particular patient should be preceded by quality improvements in diabetes care in general [15]. For this purpose, the Diabetes Quality Improvement Project (DQIP) was formed in the United States in 1997 by a coalition of private and public organizations [16]. This organization developed a set of diabetes-specific performance and outcome measures that may be used as indicators or tools for the assessment of the level of care provided within a system of management for diabetic patients. In total, seven accountability measures were adopted by the DQIP [17].

Similarly, the Belgian Diabetes Project Group extracted and presented 34 quality indicators for type 2 diabetes from various type 2 diabetes guidelines [8]. Furthermore, the working group behind this research project distinguished process indicators, which provide an indication of the quality of the process or intervention in diabetes care, from outcome indicators, which provide an indication of the quality of the outcome of a process or intervention for diabetes care [8]. The items in the Korean DQA are similar to the seven accountability measures incorporated in the DQIP and the quality indicators used by the Belgian Diabetes Project Group. As mentioned in the Introduction of the present paper, the assessment items currently in use correspond to the process indicators, and outcome indicators will be phased in the near future by HIRA [9].

A previous systematic review found that the quality improvement interventions used to enhance glycemic control in patients with type 2 diabetes significantly lowered HbA1c levels by $0.42 \%$ (95\% confidence interval, 0.29 to 0.54 ) [18]. Tricco et al. [19] reported the findings from their comprehensive systematic meta-analysis and a review of the quality improvement strategies used in diabetes care. In this systematic review, previous assessments [18] were updated and then expanded upon by including vascular risk management, monitoring of microvascular complications, and smoking cessation as well as outcomes for HbAic levels in patients with diabetes. The inclusion of cardiovascular outcomes and smoking cessation is important because it is being increasingly recognized that glycemic control alone is not adequate to prevent both the microvascular and macrovascular complications of diabetes [19]. Especially, smoking cessation is very important in the treatment of peripheral arterial disease in diabetic patients [20]. Although the Korean DQA indicators include process indicators associated with glycemic control (e.g., proportion who underwent HbAic tests), as well as vascular risk management (e.g., proportion who underwent lipid tests) and microvascular complications (e.g., proportion who underwent fundus and microalbuminuria test), the outcomes of variables such as HbAic, LDL, and blood pressure levels cannot be determined due to the lack of outcome indicators. Thus, future research will become necessary to investigate whether these outcomes are improving as the DQA indicators increase.

Currently, patients with diabetes in Korea receive treatment at various institutions, including tertiary hospitals, general hospitals, hospitals, long-term care hospitals, clinics, public health centers, branches of public health centers and health centers, and country hospitals. As of 2013, the number of subjects involved in the quality assessment was 1.89 million, among whom $60.7 \%$ were receiving treatment from clinics, $17.3 \%$ were receiving treatment from general hospitals, and $12.2 \%$ were receiving treatment from tertiary hospitals [4]. Therefore, although the quality assessment findings of the present study, obtained from a single university hospital, may not reflect the situation of national diabetes management in Korea in its entirety, the DQA results published by the HIRA only present the differences in quality assessment indicators according to each medical institution. As a result, this study has significance in that the proposed improvements for the management of diabetes were made according to the characteristics of the patients by analyzing differences in quality assessment indicators using sex, age, use of insulin, and the depart- 
ment of treatment of the patients, which the HIRA data could not determine.

An analysis of the DQA data from the hospital in the present study showed that male patients had a lower COT than that of female patients, and the insulin-treated group had a higher COT than that of the non-insulin-treated group, as well as a higher rate of taking the DCT. In terms of age, those 40 to 80 years of age accounted for the majority of visiting patients and had the highest COT, while those under 40 years had a low COT but a high rate of taking the DCT. Given that a longer duration of diabetes leads to a higher likelihood of complications [21], it is important to perform the DCT on young patients.

An analysis of the DQA data according to treatment department revealed higher proportions of performance of $\mathrm{HbAlc}$, lipid and microalbuminuria tests among the test indicators in the endo group, and lower proportions of patients who underwent lipid, fundoscopy, and microalbuminuria tests in the others group (who did not receive treatment from endocrinology specialists) than the national average from upper institutions. This group also displayed lower proportions for the test indicators, except for the HbAlc test, and higher proportions for the prescription indicators, including prescriptions with over four ingredient groups and duplicated prescriptions with the same ingredient groups.

It is considered that medical staff in our endocrinology department showed higher proportions of performance for the test indicators than the national average through the compliance with national diabetes care guidelines, continuous patient training, the establishment of own DQA indicator management system, and the 1 year notification after the performance of a test in the test indicators.

These results suggest that endocrinologists tend to combine the prevention and management of diabetes complications with measures for glycemic control, while physicians in other departments tend to only manage diabetes-related concerns, with a focus on glycemic control. This indicates that the prescription of oral hypoglycemic agents with fewer side effects and higher patient compliance and efficacy is the domain of endocrinologists.

Based on the present findings, it is necessary to determine methods to enhance the COT for male patients and patients under the age of 40 years as well as to develop measures that can maintain a high DCT rate in insulin-treated patients and patients under 40 years of age. Additionally, methods that can invigorate a joint treatment system between physicians and endocrinologists to combine glycemic control with the management of diabetes complications are needed. In terms of this joint treatment system, cooperation between endocrinologists and primary healthcare institutions in local communities needs to be considered beyond communication between these departments within large general hospitals. The potential benefits of diabetes management in this type of cooperative system have already been identified by a number of previous studies [22], but the management of diabetes in a primary care institution could still be improved by endocrinologist-supported interventions aimed at providers. Additionally, it may be important to partner generalists with diabetes specialists to enhance diabetes management in primary care settings [23].

Because $60.7 \%$ of the subjects in the present quality assessment were patients from clinics, and only $47.2 \%$ of all clinics in Korea underwent quality assessment as of 2013, it can be assumed that a substantial number of diabetic patients in Korea receive treatment from clinic-level primary healthcare institutions. While these clinics receive the highest numbers of patients who visit more than once per quarter (92.6\%) among all medical institutions, they administer fewer tests compared with the national average but are associated with more prescriptions with over four ingredient groups and duplicated prescriptions with the same ingredient group [4]. Therefore, if the abovementioned joint treatment system is reinvigorated in tertiary hospitals that treat diabetic patients, then patients who have difficulties attending outpatient treatment at tertiary hospitals can undergo DCTs and receive prescriptions of hypoglycemic agents from endocrinologists at tertiary hospitals while still receiving treatment at a nearby clinic with good access. This would be expected to improve COT and will likely result in improved management of diabetes at the national level. 


\section{KEY MESSAGE}

1. The prevention, early detection, and treatment of the complications of diabetes as well as glycemic control need to be considered for the management of diabetes.

2. Endocrinologists tend to combine the prevention and management of diabetes complications with measures for glycemic control.

3. The effective management of diabetes likely entails systematic joint treatment regimens that involve an endocrinologist.

\section{Conflict of interest}

No potential conflict of interest relevant to this article was reported.

\section{Acknowledgments}

This study was conducted using data from the Kyung Hee University Hospital Diabetes Quality Assessment system. We would like to express our gratitude to team leader Kim Jeong-ho and the staff members of the Kyung Hee University Hospital computer team for making this system. This study was supported by a grant of the Korean Health Technology R\&D Project, Ministry of Health and Welfare, Republic of Korea ( $\left.\mathrm{HC}_{15} \mathrm{C}_{33} 64\right)$.

\section{REFERENCES}

1. Organization for Economic Co-operation and Development. Cardiovascular Disease and Diabetes: Policies for Better Health and Quality of Care. Paris: OECD, 2015.

2. Statistics Korea. 2013 Cause of Death Statistics. Daejeon: Statistics Korea, 2014.

3. Ha KH, Kim DJ. Trends in the diabetes epidemic in Korea. Endocrinol Metab (Seoul) 2015;3:142-146.

4. Health Insurance Review and Assessment Service. 2013 The results for diabetes quality assessment [Internet]. Wonju: HIRA, c2013 [cited $2017 \mathrm{Feb}$ 15]. Available from: http://biz.hira.or.kr/cms/PC/noticeo1/__icsFiles/afieldfile/2015/o1/o8/PJgroKapYbhy.pdf.

5. Nathan DM, Cleary PA, Backlund JY, et al. Intensive diabetes treatment and cardiovascular disease in patients with type 1 diabetes. N Engl J Med 2005;353:2643-2653.
6. Stratton IM, Adler AI, Neil HA, et al. Association of glycaemia with macrovascular and microvascular complications of type 2 diabetes (UKPDS 35): prospective observational study. BMJ 2000;321:405-412.

7. National Center for Chronic Disease Prevention and Health Promotion (U.S.) Division of Diabetes Translation. National diabetes fact sheet, 2011 [Internet]. Atlanta (GA): US Department of Health and Human Services, Centers for Disease Control and Prevention, 2011 [cited $2017 \mathrm{Feb}$ 15]. Available from: https://www.cdc.gov/diabetes/pubs/ pdf/ndfs_2011.pdf.

8. Wens J, Dirven K, Mathieu C, Paulus D, Van Royen P; Belgian Diabetes Project Group. Quality indicators for type-2 diabetes care in practice guidelines: an example from six European countries. Prim Care Diabetes 2007;1:17-23.

9. Health Insurance Review and Assessment Service. Detailed plans of the diabetes quality assessment [Internet]. Wonju: HIRA, c2013 [cited $2017 \mathrm{Feb}$ 15]. Available from: http://biz.hira.or.kr/cms/PC/noticeo1/__icsFiles/afieldfile/2011/04/o1/Plan.hwp.

10. Health Insurance Review and Assessment Service. Followed by hypertension, diabetes evaluation results first published [Internet]. Wonju: HIRA, c2013 [cited $2017 \mathrm{Feb}$ 15]. Available from: http://download.mohw.go.kr/front_ new/modules/download.jsp?BOARD_ID $=140 \& C O N$ I $_{\text {_ }}$ $\mathrm{SEQ}=278024$ \&FILE_SEQ $=126587$.

11. Wild S, Roglic G, Green A, Sicree R, King H. Global prevalence of diabetes: estimates for the year 2000 and projections for 2030. Diabetes Care 2004;27:1047-1053.

12. Jeppesen P, Bek T. The occurrence and causes of registered blindness in diabetes patients in Arhus County, Denmark. Acta Ophthalmol Scand 2004;82:526-530.

13. Chaturvedi N, Stevens LK, Fuller JH, Lee ET, Lu M. Risk factors, ethnic differences and mortality associated with lower-extremity gangrene and amputation in diabetes: the WHO Multinational Study of Vascular Disease in Diabetes. Diabetologia 2001;44 Suppl 2:S65-S71.

14. Atkins RC. The epidemiology of chronic kidney disease. Kidney Int Suppl 2005;94:S14-S18.

15. Ibrahim JE. Performance indicators from all perspectives. Int J Qual Health Care 2001;13:431-432.

16. Fleming BB, Greenfield S, Engelgau MM, Pogach LM, Clauser SB, Parrott MA. The Diabetes Quality Improvement Project: moving science into health policy to gain an edge on the diabetes epidemic. Diabetes Care 2001;24:1815-1820. 
17. McLaughlin S. The Diabetes Quality Improvement Project. Diabetes Spectr 2000;13:5-10.

18. Shojania KG, Ranji SR, McDonald KM, et al. Effects of quality improvement strategies for type 2 diabetes on glycemic control: a meta-regression analysis. JAMA 2006;296:427-440.

19. Tricco AC, Ivers NM, Grimshaw JM, et al. Effectiveness of quality improvement strategies on the management of diabetes: a systematic review and meta-analysis. Lancet 2012;379:2252-2261.

20. Rhee SY, Kim YS. Peripheral arterial disease in patients with type 2 diabetes mellitus. Diabetes Metab J 2015;39:283-290.
21. Harrison TR, Kasper DL, Fauci AS, et al. Harrison's Principles of Internal Medicine. 19th ed. New York: McGraw-Hill Education, 2015.

22. Ziemer DC, Doyle JP, Barnes CS, et al. An intervention to overcome clinical inertia and improve diabetes mellitus control in a primary care setting: Improving Primary Care of African Americans with Diabetes (IPCAAD) 8. Arch Intern Med 2006;166:507-513.

23. Phillips LS, Ziemer DC, Doyle JP, et al. An endocrinologist-supported intervention aimed at providers improves diabetes management in a primary care site: improving primary care of African Americans with diabetes (IPCAAD) 7. Diabetes Care 2005;28:2352-1360. 
Kim YJ, et al. From who and how should you get your diabetes treated?

Supplementary Table 1. Annual numbers and percentages of the study patients classified by sex, use of insulin, age, treatment department, and duration of medication

\begin{tabular}{|c|c|c|c|c|c|c|}
\hline \multirow{2}{*}{ Variable } & \multicolumn{6}{|c|}{ Year } \\
\hline & 2009 & 2010 & 2011 & 2012 & 2013 & 2014 \\
\hline \multicolumn{7}{|l|}{ Sex } \\
\hline Male & $3,806(50.16)$ & $4,030(49.95)$ & $4,422(50.42)$ & $4,405(50.34)$ & $4,433(51.11)$ & $4,216(51.95)$ \\
\hline Female & $3,782(49.84)$ & $4,038(50.05)$ & $4,348(49 \cdot 58)$ & $4,346(49.66)$ & $4,241(48.89)$ & $3,900(48.05)$ \\
\hline \multicolumn{7}{|l|}{ Use of insulin } \\
\hline Yes & $1,839(24.24)$ & $1,929(23.91)$ & $2,037(23.23)$ & $2,026(23.15)$ & 1,902 (21.93) & $1,640(20.21)$ \\
\hline No & $5,749(75 \cdot 76)$ & $6,139(76.09)$ & $6,733(76.77)$ & $6,725(76.85)$ & $6,772(78.07)$ & $6,476(79 \cdot 79)$ \\
\hline \multicolumn{7}{|l|}{ Age, yr } \\
\hline$<40$ & $285(3.76)$ & $304(3.77)$ & $304(3.47)$ & $283(3.23)$ & $271(3.12)$ & $241(2.97)$ \\
\hline 40 to $<50$ & $745(9.82)$ & $744(9.22)$ & $747(8.52)$ & $706(8.07)$ & $681(7.85)$ & $634(7.81)$ \\
\hline 50 to $<60$ & $1,863(24.55)$ & $1,970(24.42)$ & $2,167(24.71)$ & $2,126(24.29)$ & $1,970(22.71)$ & $1,826(22.50)$ \\
\hline 60 to $<70$ & $2,761(36.39)$ & $2,834(35 \cdot 13)$ & $3,074(35.05)$ & $2,929(33 \cdot 47)$ & $2,837(32.71)$ & $2,591(31.92)$ \\
\hline 70 to $<80$ & $1,637(21.57)$ & $1,883(23.34)$ & $2,087(23.80)$ & $2,284(26.10)$ & $2,453(28.28)$ & $2,358(29.05)$ \\
\hline$\geq 80$ & $297(3.91)$ & $333(4.13)$ & $391(4.46)$ & $423(4.83)$ & $462(5 \cdot 33)$ & $466(5 \cdot 74)$ \\
\hline \multicolumn{7}{|c|}{ Treatment department } \\
\hline Endo & $1,727(22.76)$ & $1,901(23.56)$ & $2,004(22.85)$ & $2,027(23.16)$ & $2,019(23.28)$ & $2,025(24.95)$ \\
\hline Endo \& others & $2,841(37 \cdot 44)$ & $2,996(37.13)$ & $3,274(37 \cdot 33)$ & $3,217(36.76)$ & $3,112(35.88)$ & $2,708(33 \cdot 37)$ \\
\hline Others & $3,020(39.80)$ & 3,171 (39.30) & $3,492(39.82)$ & $3,507(40.08)$ & $3,543(40.85)$ & $3,383(41.68)$ \\
\hline \multicolumn{7}{|c|}{ Duration of medication, yr } \\
\hline$<3$ & $2,315(30.51)$ & $2,356(29.20)$ & $2,497(28.47)$ & $2,216(25 \cdot 32)$ & $1,957(22.56)$ & $1,811(22.31)$ \\
\hline $3-5$ & $2,173(28.64)$ & $2,323(28.79)$ & $2,405(27.42)$ & $2,386(27.27)$ & $2,410(22.78)$ & $2,158(26.59)$ \\
\hline$\geq 6$ & $3,100(40.85)$ & $3,389(42.01)$ & $3,868(42.01)$ & $4,149(47 \cdot 41)$ & $4,307(49.65)$ & $4,147(51.10)$ \\
\hline Total & $7,588(100)$ & $8,068(100)$ & $8,770(100)$ & $8,751(100)$ & $8,674(100)$ & $8,116(100)$ \\
\hline
\end{tabular}

Values are presented as number (\%). 


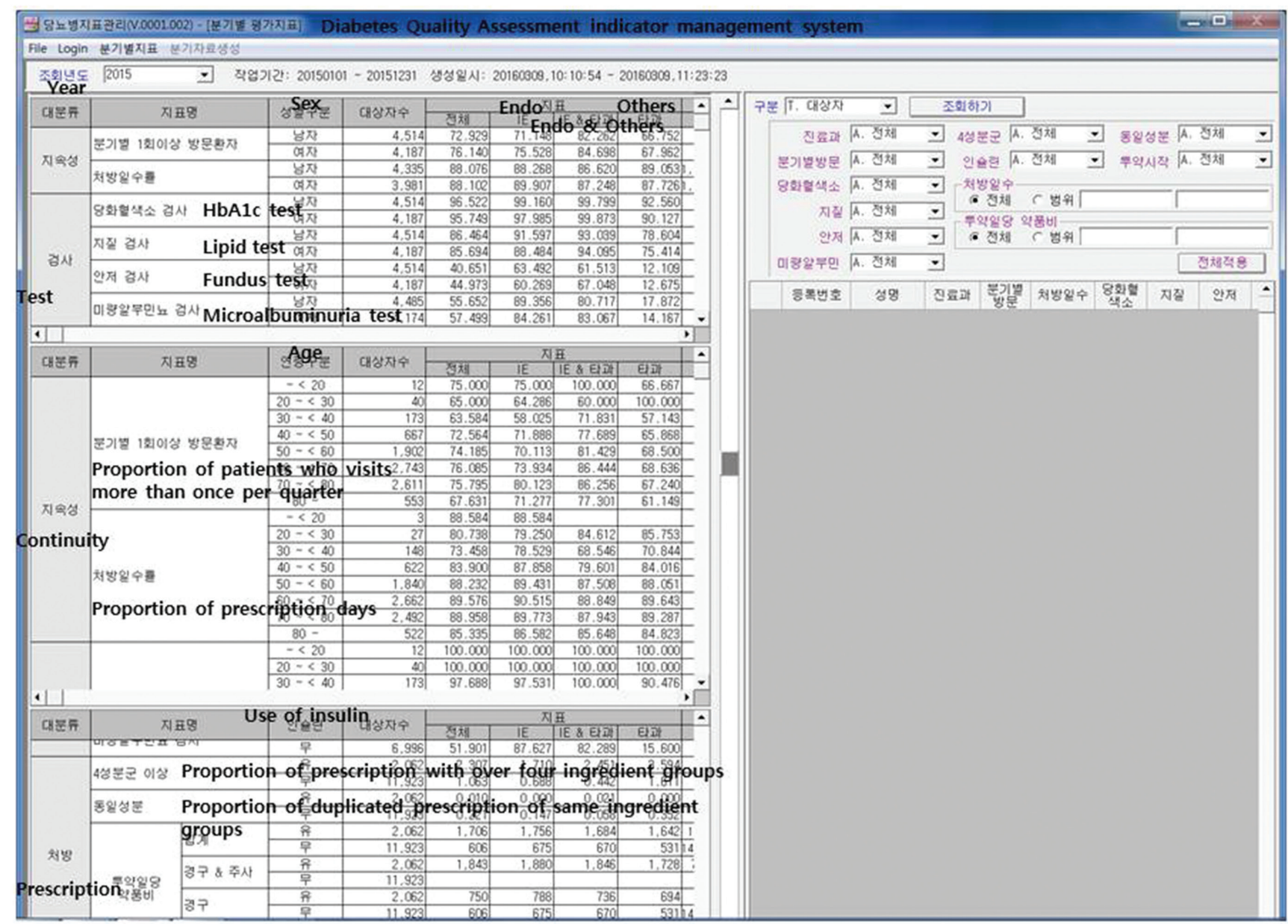

Supplementary Figure 1. The diabetes quality assessment indicator management system at Kyung Hee University Hospital. This system consists of three primary categories of indicators: continuity, test, and prescription. Each indicator is distinguished by the treatment department, sex, age, use of insulin, and duration of the administration of oral hypoglycemic agents and insulin. The total number of subjects, number of subjects who meet the indicator, and the formula for calculating the indicators are shown. HbA1c, glycated hemoglobin. 


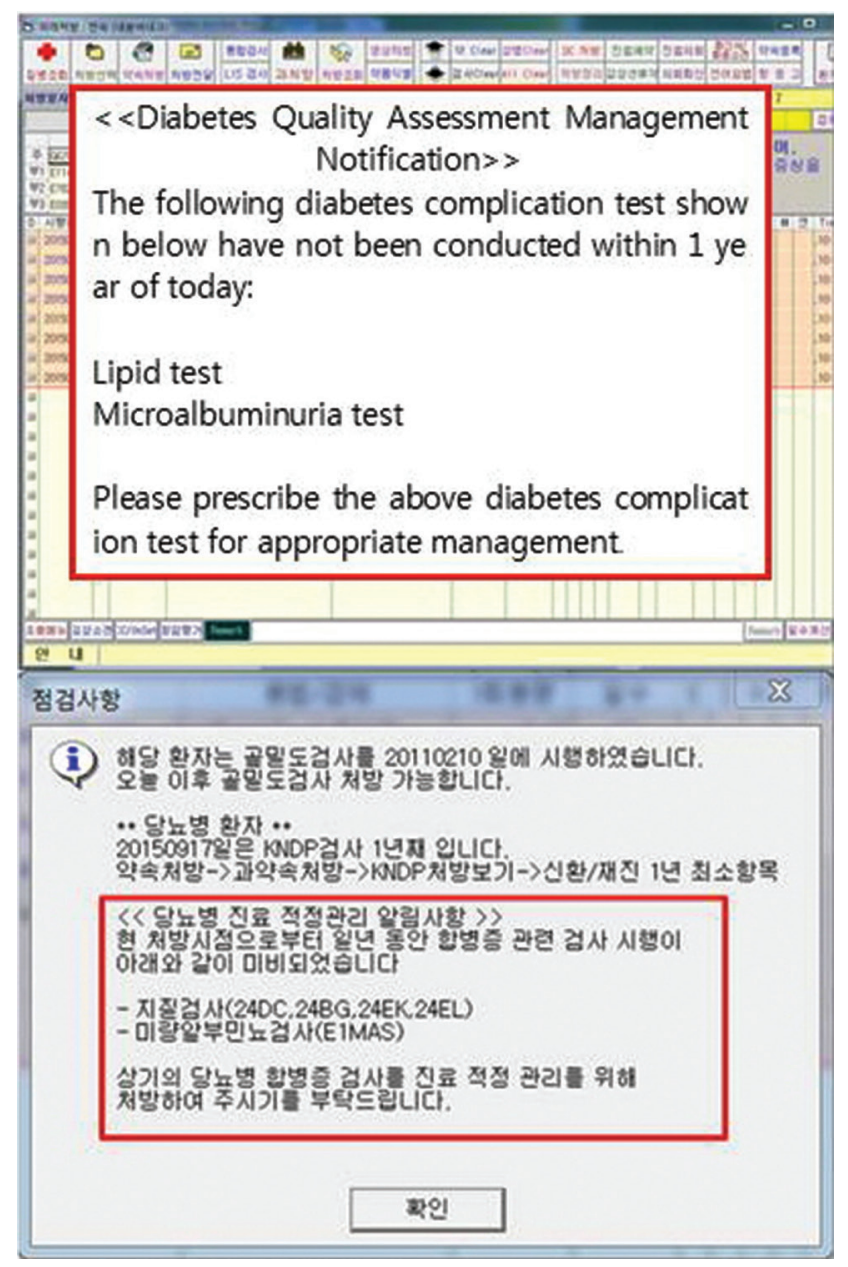

Supplementary Figure 2. The diabetes treatment appropriateness management notification system of Kyung Hee University Hospital. This system is an electronic prescription program used when prescribing medications and examinations for diabetic patients in an outpatient setting. A test notification function was added to this system; it notifies physicians of the timing of prescriptions for the diabetes complication tests through a notification window, 1 year after the $\mathrm{HbAic}$, lipid, fundus, and microalbuminuria tests. 